\title{
Dissecting aneurysms of the vertebral artery - angiographic patterns at the dissecting site on balloon test occlusion
}

\author{
Yutaka Kai • Jun-ichiro Hamada • Motohiro Morioka • \\ Yuki Ohmori • Masaki Watanabe • Teruyuki Hirano • \\ Takayuki Kawano $\cdot$ Shigetoshi Yano $\cdot$ Jun-ichi Kuratsu
}

Received: 16 December 2010 / Accepted: 29 November 2011 / Published online: 15 December 2011

(C) The Author(s) 2011. This article is published with open access at Springerlink.com

\begin{abstract}
Introduction At present, the risk of future hemorrhage or ischemic insult from vertebral artery (VA) dissection cannot be estimated from available imaging data. We investigated the relationship between symptoms and the angiographic patterns of the dissecting site on balloon test occlusion (BTO) to develop guidelines for clinical decision-making. Methods We retrospectively reviewed 18 patients with unilateral VA dissection who presented with subarachnoid hemorrhage (SAH) or cerebral infarction. We analyzed the angiographic findings at the dissecting site on contralateral VA angiograms during BTO of the affected VA, classified the angiographic patterns into two types, and compared the symptoms they presented.

Results Patients with dissection opacified from the distal to the proximal side are more likely to present with cerebral
\end{abstract}

Y. Kai $(\bowtie)$

Department of Cerebrovascular and Acute Coronary Syndrome, Graduate School of Medical Sciences, Kumamoto University, 1-1-1 Honjo,

Kumamoto, Kumamoto 860-8556, Japan

e-mail: ykai@fc.kuh.kumamoto-u.ac.jp

Y. Kai $\cdot$ M. Morioka $\cdot$ Y. Ohmori $\cdot$ T. Kawano $\cdot$ S. Yano $\cdot$

J.-i. Kuratsu

Department of Neurosurgery,

Graduate School of Medical Sciences, Kumamoto University,

Kumamoto, Japan

J.-i. Hamada

Department of Neurosurgery,

Graduate School of Medical Sciences, Kanazawa University,

Kanazawa, Japan

M. Watanabe $\cdot$ T. Hirano

Department of Neurology, Graduate School of Medical Sciences, Kumamoto University,

Kumamoto, Japan infarction than SAH. Conversely, patients with dissection opacified from the proximal to the distal side had a significantly higher incidence of SAH.

Conclusions Angiographic findings at the dissecting site on contralateral VA angiograms during BTO of the affected VA are helpful for considering the treatment and prognosis of patients with VA dissecting aneurysms.

Keywords Angiography B Balloon test occlusion ·

Dissecting aneurysm $\cdot$ Vertebral artery

$\begin{array}{ll}\text { Abbreviations } \\ \text { SAH } & \text { Subarachnoid hemorrhage } \\ \text { VA } & \text { Vertebral artery } \\ \text { BTO } & \text { Balloon test occlusion } \\ \text { MRI } & \text { Magnetic resonance imaging } \\ \text { MRA } & \text { MR angiographs } \\ \text { PICA } & \text { Posterior inferior cerebellar artery } \\ \text { PCA } & \text { Posterior cerebral artery } \\ \text { GOS } & \text { Glasgow outcome scale } \\ \text { MD } & \text { Moderate disability }\end{array}$

Introduction

Increased awareness and better diagnostic methods have led to an increase in the number of spontaneous, diagnosed, and treated intradural arterial dissections. It has been proposed that patients initially suffering from subarachnoid hemorrhage (SAH) should undergo early repair of the aneurysm by open surgery or obliteration of the aneurysm by endovascular procedures [1-4]. Rebleeding most often occurs during the acute phase following aneurysmal rupture and is thought to be the strongest prognostic indicator of a poor outcome. On the other 
hand, as unruptured vertebral artery (VA) dissection follows a relatively benign clinical course, conservative treatment has been advocated [5-9].

Many previous studies have provided initial angiographic findings on VA dissections, e.g., the pearl-and-string or the string sign, tapered occlusion, formation of a fusiform or saccular aneurysm, the appearance of a double lumen, and retention of contrast material in the late phase [1-13]. Among their anterograde diagnostic angiographic appearances, dilating lesions and a pearl-and-string structure predominated among hemorrhagic dissecting lesions [4]. However, the risk of future hemorrhage or ischemic insults from VA dissection cannot be estimated from currently available imaging data.

To our knowledge, there have been no studies on the angiographic appearance of the dissecting site on contralateral VA angiograms obtained during balloon test occlusion (BTO) of the involved VA. In the current investigation, we focused on the relationship between symptoms and the retrograde angiographic patterns of the dissecting site during BTO of the affected VA to identify prognostic factors in patients with spontaneous dissection of the VA.

\section{Materials and methods}

This is a retrospective review of imaging data on consecutively admitted patients with unilateral VA dissecting aneurysms treated at our institution between April 1998 and March 2003. Patients with SAH underwent four-vessel angiography. Patients with ischemic symptoms underwent magnetic resonance imaging (MRI). MRI was with conventional spin-echo techniques with $\mathrm{T} 1$ weighting; proton density imaging and T2-weighted MRI were performed in patients with ischemic symptoms. MR angiographs (MRA) were acquired in all patients. Patients with progressive ischemic symptoms or enlargement of the dissection site during the follow-up period underwent conventional angiography.

A 6-Fr guiding catheter (Medikit, Tokyo, Japan) was inserted to the affected VA. A heparin intravenous bolus of 5,000 units was usually sufficient to obtain an activated clotting time of about $250 \mathrm{~s}$. Heparinization was used in all patients even those with SAH at onset. All patients underwent diagnostic angiography followed by 15 -min occlusion tolerance test using a nondetachable silicon balloon (Equinox, Micro Therapeutics, Inc. Irvine, CA, USA) to occlude the parent VA just proximal to the dissecting aneurysm. Neurological findings were recorded during BTO. If neurological deficits such as consciousness disturbance or focal neurological deficits were recognized BTO was stopped immediately, otherwise it was continued for $15 \mathrm{~min}$. During BTO of the affected VA, continuous-mode angiography (20 frames per second) of the contralateral VA was performed.

We studied findings on the dissecting site on contralateral vertebral angiograms during BTO. Only aneurysms that were located between the origin of the posterior inferior cerebellar artery (PICA) and the VA junction, or located in the VA without definite PICA involvement were analyzed to exclude the influence of retrograde flow into the PICA from the contralateral VA. Patients whose dissecting site was located proximal to the PICA were excluded because retrograde flow from the contralateral VA was directly into the ipsilateral PICA.

The angiographic pattern of retrograde filling into the dissecting site during BTO was classified into two types and their symptoms were compared. In type A, the dissection was visualized from the distal to the proximal side (Fig. 1); in type $\mathrm{B}$, opacification was observed from the proximal to the distal side (Fig. 2).

All patients and/or their families gave prior informed consent for BTO. The study protocol was approved by the clinical investigation committee of Kumamoto University. Our basic treatment policy for ruptured VA dissecting aneurysms has been reported elsewhere $[14,15]$. Patients who tolerated BTO underwent interventional surgery using detachable coils to trap the dissecting site. For trapping, we used the double microcatheter technique that facilities the simultaneous embolization of both proximal and distal borders of the dissecting segment [15]. Patients who did not tolerate BTO underwent VA trapping with the VA posterior cerebral artery (PCA) bypass procedure using a radial artery graft [14]. In patients with unruptured VA dissecting aneurysm and no enlargement of the dissecting site or new symptoms, we assessed additional treatments to prevent bleeding. The management outcome was evaluated using the Glasgow outcome scale (GOS) at 6-month follow-up.

\section{Results}

Between April 1998 and March 2003, 65 patients presented with SAH or ischemic symptoms from a dissection in the unilateral VA. Of the 65 aneurysms, 15 were located between the origin of the PICA and the VA junction, three were in the VA without definite PICA involvement. These 18 aneurysms were the focus of our retrospective study. Angiographically, at the time of diagnosis, the 18 aneurysms appeared as a sausage-like swelling $(n=6)$, pearl- and/or string sign $(n=8)$, or as retention of contrast material $(n=4)$.

The age of the 18 patients ranged from 43 to 67 years (mean 44.5 years); 10 presented with SAH and eight with cerebral infarction. Preoperatively, of the 10 patients with $\mathrm{SAH}$, two each were recorded as grade I and grade II and six as grade III on the Hunt and Hess score. 

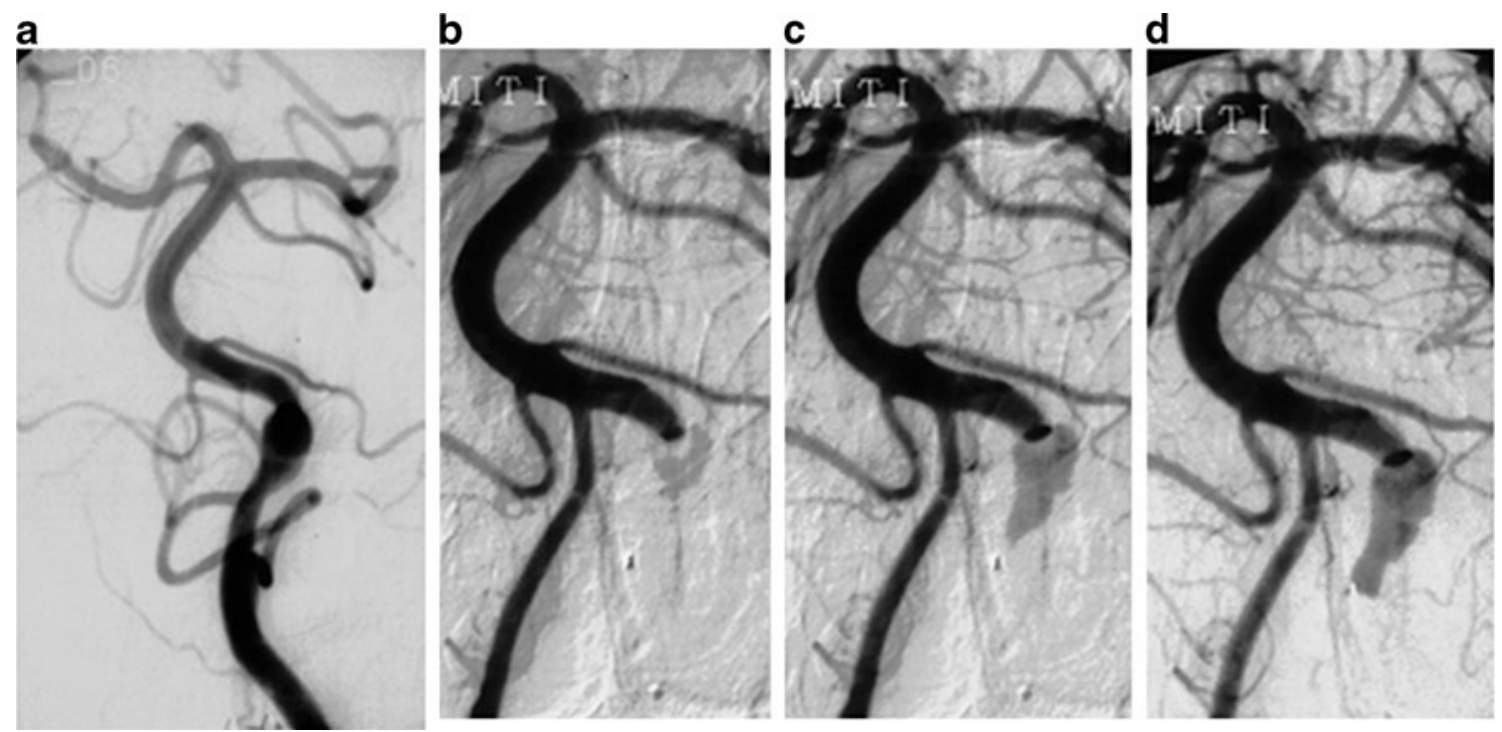

Fig. 1 Type A dissecting aneurysm. a DSA of the left vertebral artery showing a fusiform dilation. $\mathbf{b}, \mathbf{c}, \mathbf{d}$ Consecutive angiograms of the right vertebral artery during BTO showing that the site of dissection is opacified distal to proximal

The interval between the onset of symptoms and BTO varied from $6 \mathrm{~h}$ to 16 days; the mean interval was 3.2 days in the patients with SAH and 9.7 days in those with ischemic symptoms. There were no significant complications attributable to BTO; no complications related to thrombosis or rebleeding occurred during BTO. Our classification of the 18 aneurysms based on their angiographic pattern at the site of dissection showed that 12 were of type A and six of type B. Of the 12 patients with type A, four (33\%) presented with SAH and eight $(67 \%)$ with cerebral infarction. All six type B patients suffered SAH (Table 1) indicating that patients with type A dissections tended to present with cerebral infarction rather than SAH. On the other hand, patients with type B had a significantly higher rate of SAH as the initial symptom by the $\chi^{2}$ test $(p=0.01)$.

Of the 18 patients, 11 were treated, i.e., all 10 patients with SAH (type A, $n=4$; type $\mathrm{B}, n=6$ ) and one patient with cerebral infarction (type A) underwent treatment. We treated the patient with cerebral infarction because his ischemic symptoms worsened instead of antiplatelet therapy on day 16. All 11 patients were treated immediately after BTO. In 10 patients (SAH, $n=9$, cerebral infarction, $n=1$ ), we trapped the VA dissecting site using detachable coils. One patient with SAH (type A) underwent VA trapping and a VA-PCA bypass using a radial artery graft. There were two patients with neurological deficits after treatment. In one, a

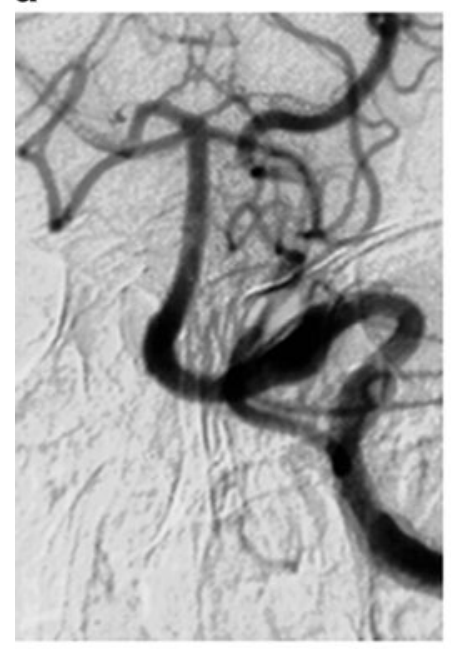

b

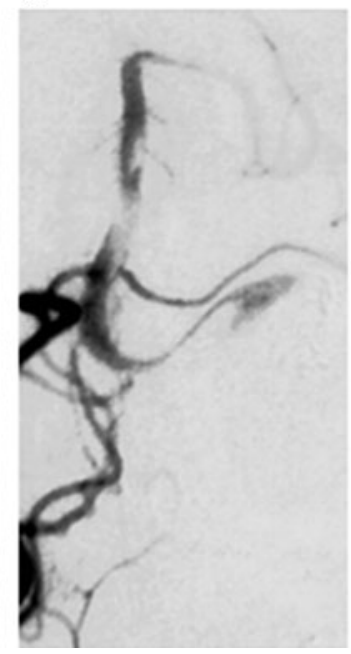

C

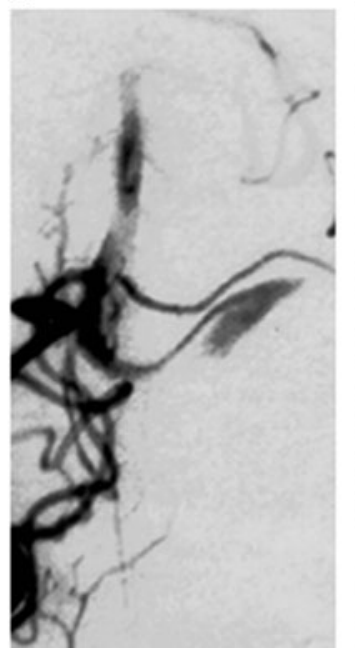

d

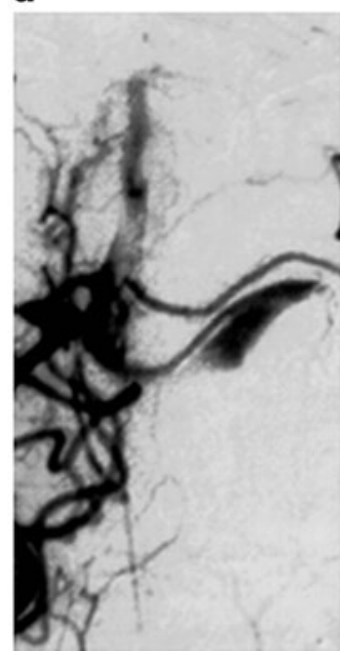

Fig. 2 Type B dissecting aneurysm. a DSA of the left vertebral artery showing a fusiform dilation. b, c, d Consecutive angiograms of the right vertebral artery during BTO showing that the site of dissection is opacified proximal to distal 
Table 1 Angiographic patterns at the dissecting site and symptoms on balloon test occlusion

\begin{tabular}{lcc}
\hline Type & $\begin{array}{l}\text { Subarachnoid } \\
\text { hemorrhage (\%) }\end{array}$ & Cerebral infarction \\
\hline Type A $(n=12)$ & $4(33)$ & $8(67)$ \\
Type B $(n=6)^{*}$ & $6(100)$ & $0(0)$ \\
Total $(n=18)$ & $10(56)$ & $8(44)$ \\
\hline
\end{tabular}

Values in parentheses are percentages

${ }^{*} p=0.01$, significant difference; $\chi^{2}$ test

mild ataxia was recognized; it resolved at 6 months posttreatment and he manifests moderate disability (MD). The other patient transiently exhibited mild lateral medullary syndrome; his symptoms improved at 3 months post-treatment. Two patients required angioplasty due to vasospasm; their mild hemiparesis subsequently improved. At 6 months after treatment, 10 patients manifested good recovery and one MD on the GOS. There were no patients with recurrent hemorrhage during post-treatment follow-up of 2 years. We treated

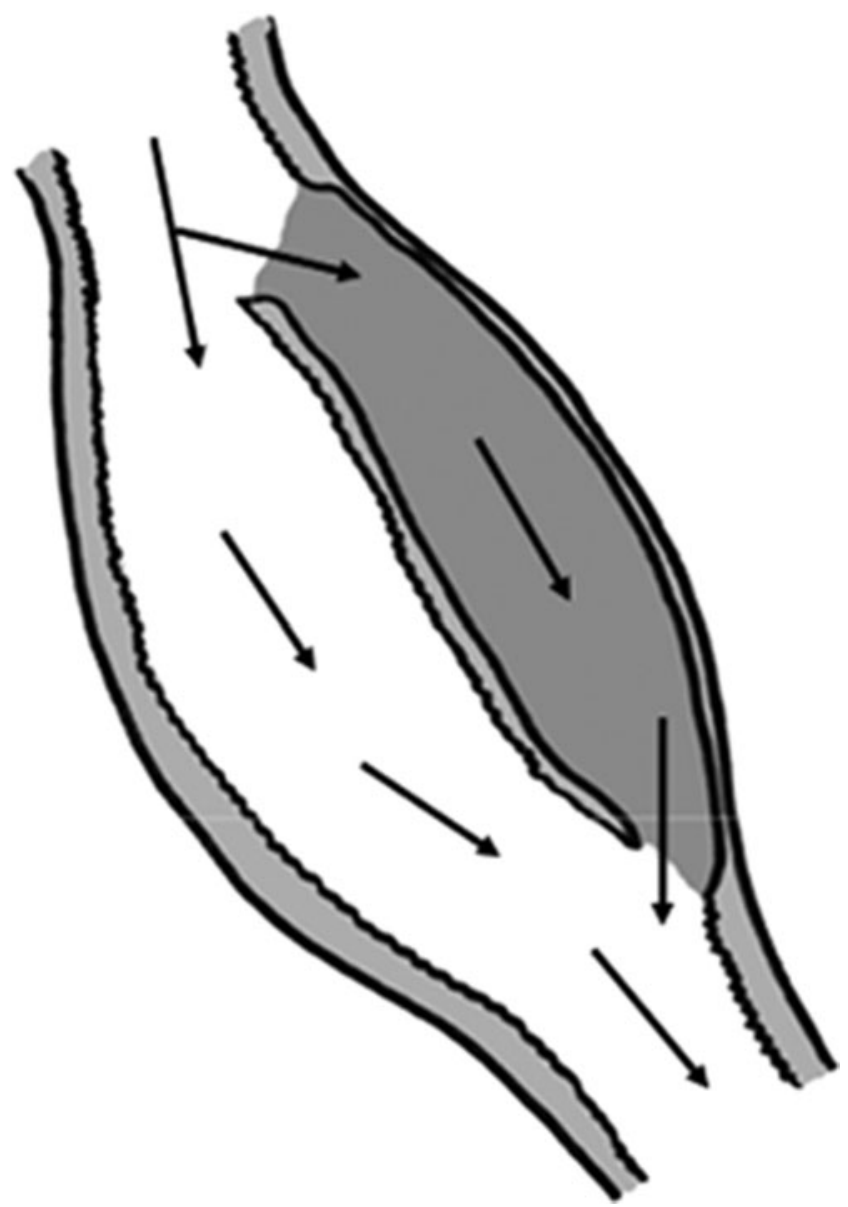

Fig. 3 Illustration of a type A dissecting aneurysm. The gray area represents the pseudolumen. Arrows demonstrate the flow of blood through the true lumen and the pseudolumen seven patients with type A cerebral infarction conservatively; they suffered no additional neurological deficits during the follow-up period of 2 years. Based on the GOS, at 6 months after the insult, all seven manifested good recovery.

\section{Discussion}

Although it can result in complications, cerebral angiography is currently the method of choice for a radiological diagnosis of VA dissecting aneurysms. The major documented angiographic findings associated with VA dissecting aneurysms are irregular stenosis with or without focal dilation, known as the "pearland-string sign," and occlusion of the affected vessel [1-13]. These are indirect signs suggestive of a dissecting aneurysm. Delayed retention of contrast medium in the partially thrombosed pseudolumen is considered a relatively reliable sign supporting this diagnosis and although rarely encountered, an angiographic "double lumen" is a definitive diagnostic sign.

Conventional angiography, while useful for the diagnosis of dissecting aneurysms, places patients under stress and may

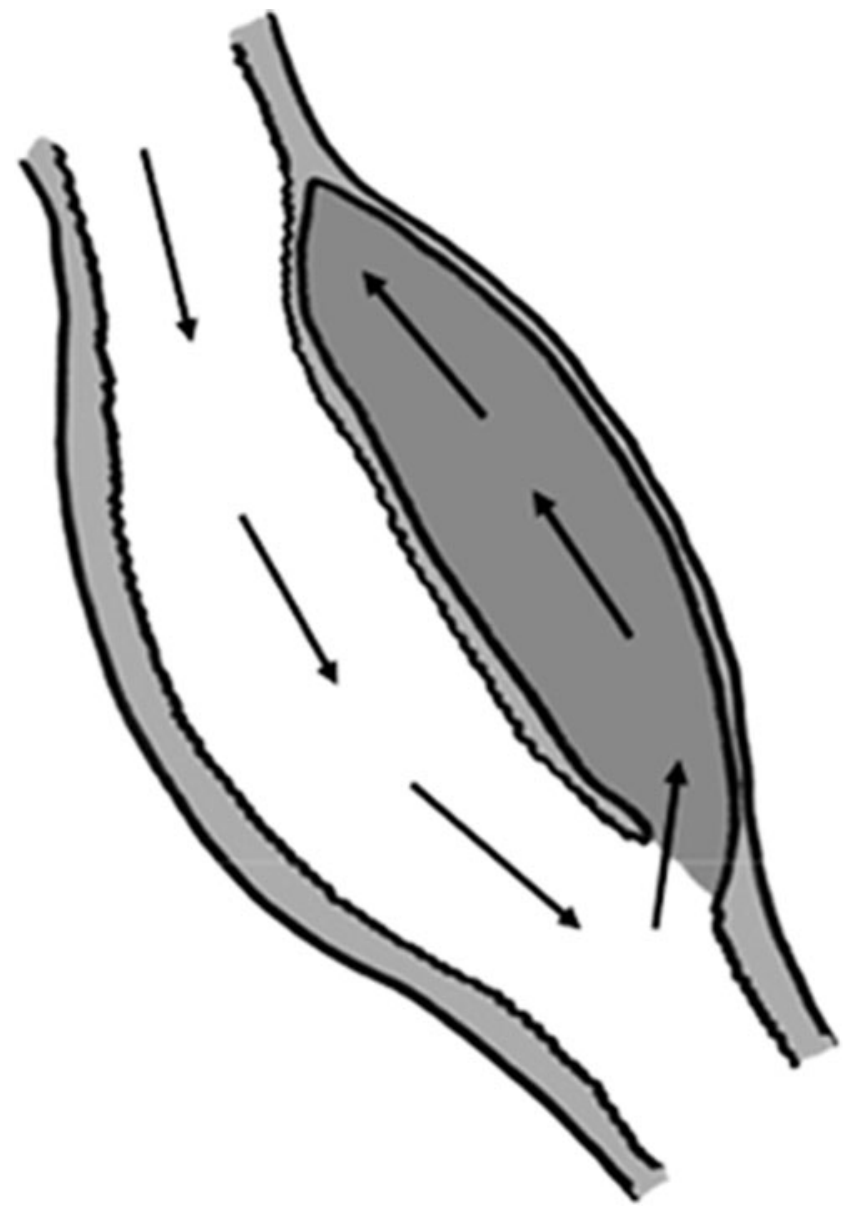

Fig. 4 Illustration of a type B dissecting aneurysm. The gray area represents the pseudolumen. Arrows demonstrate the flow of blood through the true lumen and the pseudolumen 
elicit complications. Because MRI and MRA studies are noninvasive, they can be performed repeatedly to monitor the site of dissection for changes over time. On T1-weighted- and fluid-attenuated inversion recovery MRI scans, intraluminal VA hematomas can be detected and the pearl-and-string and the double lumen sign are characteristic MRA findings of VA dissecting aneurysms [16-18]. On 3D contrast-enhanced images, a double lumen at the site of dissection is visualized [18]. Basiparallel anatomical scanning MRI, a technique that reveals the surface contour of the intradural vertebrobasilar artery, aids in the evaluation of the clinical course of unruptured VA dissections [12, 19, 20].

Most initial angiograms of unruptured VA dissecting aneurysms demonstrate a stenotic pattern including an occlusive stricture [5-9]. Mizutani et al. presented a series of 42 patients with ruptured VA dissecting aneurysms; 30 suffered subsequent hemorrhages [2]. In their series, the most common and typical angiographic findings were, in order, an irregular fusiform appearance, alternating irregular stenotic and dilated segments, and retention of contrast medium in the late phase. However, there was no significant correlation between the angiographic appearance of the aneurysms and their subsequent rupture.

Yamada et al. reported that the pearl-and-string appearance of VA dissecting aneurysms was a significant prognostic factor for a poor outcome [3]. They postulated that ruptured pearl-and-string aneurysms may be the result of a dissection in the wall of the lesion that reaches the adventitia, and that this may increase their tendency to bleed, resulting in repeated hemorrhages and poor outcomes. In lesions with stenotic or occlusive strictures, the dissecting hematoma may not reach the adventitia and this may explain why patients with this type of lesion had good outcomes. Therefore, they suggest that patients with angiographic features of a pearl-and-string stricture may be candidates for aggressive attempts to prevent rebleeding in the acute period.

Mizutani et al. investigated the pathological mechanism(s) and precise $3 \mathrm{D}$ structure of nine cerebral ruptured dissecting aneurysms and their clinical course [21]. According to their findings, the primary mechanism by which a cerebral dissecting aneurysm is created is the sudden disruption of the internal elastic lamina with the plane of dissection extending through the media. The majority of aneurysms have one entrance into the pseudolumen (entry-only type) and this type is associated with an unstable clinical course. Some cerebral dissecting aneurysms have both an entrance and exit (entry-exit type) and maintain a constant flow of blood through the pseudolumen; these aneurysms are clinically more stable than the entry-only type.

Our type A (Fig. 3) dissecting aneurysms are consistent with the entry-exit type of Mizutani et al. while the type B (Fig. 4) aneurysms are compatible with their entry-only type. In the course of our 2-year follow-up, seven patients with type A cerebral infarction were treated conservatively, they suffered no further hemorrhage or ischemic attacks. One-third of the 12 patients with type A presented with $\mathrm{SAH}$ and was at low risk for bleeding. All six type B patients manifested $\mathrm{SAH}$, indicating that patients with type A dissections tended to present with cerebral infarction rather than $\mathrm{SAH}$. On the other hand, patients with type B had a significantly higher rate of SAH as the initial symptom by the $\chi^{2}$ test $(p=0.01)$.

\section{Conclusions}

Patients whose dissection on contralateral angiograms of the VA during BTO was visualized from the proximal to the distal side had a significantly higher incidence of SAH as the initial symptom. Conversely, patients with a dissection opacified from the distal to the proximal side tended to present with cerebral infarction rather than SAH. These findings are of use when considering the prognosis and optimal treatment of VA dissecting aneurysms. Further studies with larger populations are underway to test our preliminary findings and to provide guidelines for clinical decision making.

Conflict of interest We declare that we have no conflict of interest.

Open Access This article is distributed under the terms of the Creative Commons Attribution Noncommercial License which permits any noncommercial use, distribution, and reproduction in any medium, provided the original author(s) and source are credited.

\section{References}

1. Kitanaka C, Sasaki T, Eguchi T, Teraoka A, Nakane M, Hoya K (1994) Intracranial vertebral artery dissections: clinical, radiological features, and surgical considerations. Neurosurgery 34:620-627

2. Mizutani T, Aruga T, Kirino T, Miki Y, Saito I, Tsuchida T (1995) Recurrent subarachnoid hemorrhage from untreated ruptured vertebral dissecting aneurysms. Neurosurgery 36:905-913

3. Yamada M, Kitahara T, Kurata A, Fujii K, Miyasaka Y (2004) Intracranial vertebral artery dissection with subarachnoid hemorrhage: clinical characteristics and outcomes in conservatively treated patients. J Neurosurg 101:25-30

4. Yamaura A, Watanabe Y, Saeki N (1990) Dissecting aneurysms of the intracranial vertebral artery. J Neurosurg 72:183-188

5. de Bray JM, Penisson-Besnier I, Dubas F, Emile J (1997) Extracranial and intracranial vertebrobasilar dissections: diagnosis and prognosis. J Neurol Neurosurg Psychiatry 63:46-51

6. Kitanaka C, Tanaka JI, Kuwahara M, Teraoka A, Sasaki T, Takakura K, Tanaki J (1994) Nonsurgical treatment of unruptured intracranial vertebral artery dissection with serial follow-up angiography. J Neurosurg 80:667-674

7. Mokri B, Houser OW, Sandok BA, Piepgras DG (1988) Spontaneous dissections of the vertebral arteries. Neurology 38:880-885

8. Pozzati E, Padovani R, Fabrizi A, Sabattini L, Gaist G (1991) Benign arterial dissections of the posterior circulation. J Neurosurg 75:69-72 
9. Yoshimoto Y, Wakai S (1997) Unruptured intracranial vertebral artery dissection: clinical course and serial radiographic imagings. Stroke 28:370-374

10. Pozzati E, Andreoli A, Limoni P, Casmiro M (1994) Dissecting aneurysms of the vertebrobasilar system: study of 16 cases. Surg Neurol 41:119-124

11. Provenzale JM, Morgenlander JC, Gress D (1996) Spontaneous vertebral dissection: clinical, conventional angiographic, CT, and MR findings. J Comput Assist Tomogr 20:185-193

12. Takada H, Hyogo T, Kataoka T, Hayase K, Nakamura H (2006) Diagnosis of vertebral artery dissection by basi-parallel anatomical scanning (BPAS) MRI. Interv Neuroradiol 12:129-132

13. Yonas H, Agamanolis D, Takaoka Y, White RJ (1977) Dissecting intracranial aneurysms. Surg Neurol 8:407-415

14. Hamada J, Kai Y, Morioka M, Yano S, Todaka T, Ushio Y (2003) Multimodal treatment of ruptured dissecting aneurysms of the vertebral artery during the acute stage. J Neurosurg 99:960966

15. Kai Y, Hamada JI, Morioka M, Todaka T, Mizuno T, Ushio Y (2003) Endovascular coil trapping for ruptured vertebral artery dissecting aneurysms by using double microcatheter technique in the acute stage. Acta Neurochir (Wien) 145:447-451
16. Hosoya T, Adachi M, Yamaguchi K, Haku T, Kayama T, Kato T (1999) Clinical and neuroradiological features of intracranial vertebrobasilar artery dissection. Stroke 30:1083-1090

17. Naito I, Iwai T, Sasaki T (2002) Management of intracranial vertebral artery dissections initially presenting without subarachnoid hemorrhage. Neurosurgery 51:930-937

18. Nakagawa K, Touho H, Morisako T, Osaka Y, Tatsuzawa K, Nakae H, Owada K, Matsuda K, Karasawa J (2000) Long-term follow-up study of unruptured vertebral artery dissection: clinical outcomes and serial angiographic findings. J Neurosurg 93:19-25

19. Nagahata M, Abe Y, Ono S, Hosoya T, Uno S (2005) Surface appearance of the vertebrobasilar artery revealed on basiparallel anatomic scanning (BPAS)-MR imaging: Its role for brain MR examination. AJNR Am J Neuroradiol 26:2508-2513

20. Nagahata M, Manabe H, Hasegawa S, Takemura A (2006) Morphological change of unruptured vertebral artery dissection on serial MR examinations. Evaluation of the arterial outer contour by basi-parallel anatomical scanning (BPAS)-MRI. Interv Neuroradiol 12:133-136

21. Mizutani T, Kojima H, Asamoto S, Miki Y (2001) Pathological mechanism and three-dimensional structure of cerebral dissecting aneurysms. J Neurosurg 94:712-717 